\title{
Cancer cell invasion of brain tissue: guided by a prepattern?
}

\author{
MICHAEL WURZEL $\dagger$, CARLO SCHALLER $\ddagger$ MATTHIAS SIMON $\ddagger$ and ANDREAS DEUTSCH $\dagger *$ \\ $†$ Center for High Performance Computing, Dresden University of Technology D-01062, Dresden, Germany \\ $\ddagger$ Department of Neurosurgery, Medical Center, University of Bonn, Bonn, Germany
}

(Received 2 August 2004; in final form 29 November 2004)

\begin{abstract}
The malignant brain tumour Glioblastoma multiforme (GBM) displays a highly invasive behaviour. Spreading of the malignant cells appears to be guided by the white matter fibre tracts within the brain. In order to understand the global growth process we introduce a lattice-gas cellular automaton model which describes the local interaction between individual malignant cells and their neighbourhood. We consider interactions between cells (brain cells and tumour cells) and between malignant cells and the fibre tracts in the brain, which are considered as a prepattern. The prepattern implies persistent individual cell motion along the fibre structure. Simulations with the model show that only the inclusion of the prepattern results in invading tumour and growing tumour islets in front of the expanding tumour bulk (i.e. the growth pattern observed in clinical practice). Our results imply that the infiltrative growth of GBMs is, in part, determined by the physical structure of the surrounding brain rather than by intrinsic properties of the tumour cells.
\end{abstract}

Keywords: Glioblastoma; White matter; Fibre tracts; Lattice-gas cellular automation; Expansive growth; Growth by invasion

\section{Introduction}

Glioblastomas (Glioblastoma multiforme WHO grade IV, GBM) account for $50-60 \%$ of all primary brain tumours and up to $30 \%$ of all intracranial neoplasms [1]. A typical GBM consists of an irregularly shaped and well vascularized solid tumour mass with a necrotic core (figure 1). There is no sharp boundary between the tumour and the parenchyma of the brain. Tumour cells infiltrate the surrounding brain tissue and can be found at quite a distance away from the main tumour mass in areas completely inconspicuous by routine histopathological analysis (see Chicoine and Silbergeld [2] and Silbergeld and Chicoine [3]).

Standard therapy for GBMs consists of surgical removal of all visible tumour (gross total resection) followed by radiotherapy administered to the tumour bed (site of the original tumour after it is surgically removed) maintaining a safety margin of $2-3 \mathrm{~cm}$. However, unfortunately all of these GBMs will recur and more than $75 \%$ within the radiation fields [4]. Chemotherapy is often used after tumour recurrence [5]. Placement of biodegradable wafers containing a chemotherapeutic agent at the time of surgery may confer a modest survival benefit [6]. In spite of all the progress in recent decades, average survival in unselected neurosurgical series does not exceed 12 months [7].

Conceptually, GBMs grow by expansion and by invasion [8]. Expansive growth can be localized to areas with high blood supply and is characterized by mitosis and volume increase of single malignant cells. Due to tumour expansion, the brain undergoes deformation, but the local topology (neighbourhood) of the intact brain tissue is not destroyed. Growth by invasion is defined by the movement of isolated single malignant cells invading and alternately destroying the brain parenchyma. Experimental and clinical data suggest that malignant cells move faster in the white than in the grey matter [8-10]. The white matter consists of axonal fibre tracts providing physically permissive tracts for tumour cells to migrate along the fibres. These white matter tracts interconnect various ipsi- and contralateral (on the same and on the opposite side, respectively) regions of the brain, quite in accordance with the patterns of GBM spread commonly observed in clinical practice. On the contrary, the structure of the grey matter is more complex and may actually constitute a physical barrier for moving cells.

*Corresponding author. E-mail: deutsch@zhr.tu-dresden.de

Journal of Theoretical Medicine

ISSN 1027-3662 print/ISSN 1607-8578 online @ 2005 Taylor \& Francis Group Ltd

http://www.tandf.co.uk/journals

DOI: $10.1080 / 1027366042000334144$ 


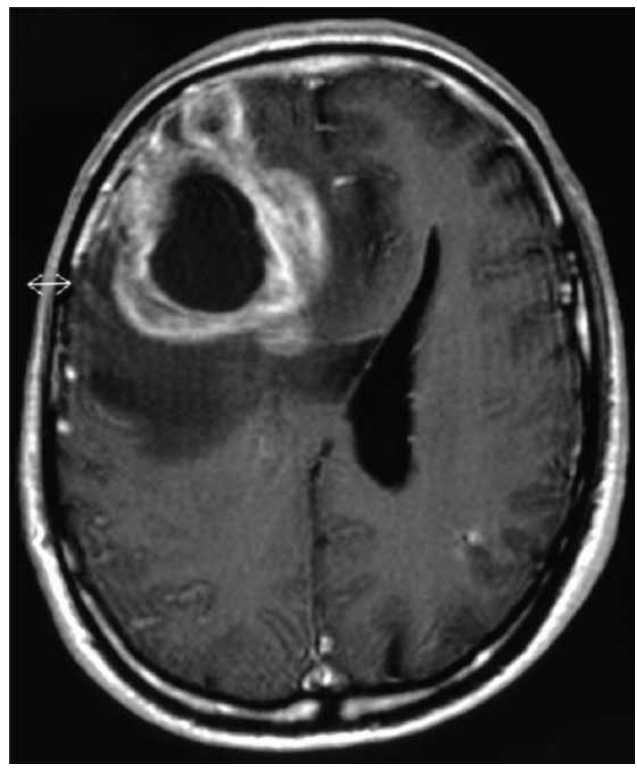

Figure 1. Glioblastoma multiforme located in the frontal lobe. Axial magnetic resonance imaging (MRI) shows a necrotic core surrounded by contrast enhancing, almost ring-shaped viable tumour tissue and hypointense (low in signal) white matter edema. Malignant cells can be found throughout the edematous white matter and even beyond [3].

There is strong evidence that the movement of tumour cells is controlled by adhesion: "trails" in the brain used by malignant glial cells and neural stem cells are probably similar [11]. Malignant glial cells may fall back into stem cell behaviour and follow tracts defined by neural cells and blood vessels [11-13]. Furthermore, genetic alterations observed in malignant cells affect cell movement and adhesion [12].

In order to understand the basic principles of GBM growth it is useful to create a mathematical framework that models proliferation and invasion. The direction of movement may be affected by the gradient of nutritive and other molecular signals (chemotaxis) and could also be a directed response to a gradient of adhesion (haptotaxis). Other processes that must be taken into account are proteolysis and contact inhibition. Subpopulations may be used to model different behaviours of malignant cells. Analysing travelling wave solutions of partial differential equations (PDEs) provides information about the infiltrative behaviour and the influence of the respective processes [14-17]. When describing only the malignant cell population, the GBM development can be simulated in a first approximation by a simple reactiondiffusion equation $[18,19]$. Space-dependent diffusion coefficients allow modeling of the influence of the heterogeneous distribution of white and grey matter on tumour cell movement [20-24].

Use of a hybrid cellular automaton takes into account both the discreteness of cells and the continuous concentrations of signals and nutrients (CA; see Moreira and Deutsch [25]) for an overview about cellular automata used in tumour modelling). Hybrid CA/PDE models may be used to describe early tumour growth and the invasive behaviour of malignant cells controlled by chemotaxis, haptotaxis and interaction with blood vessels [26-30].

None of the mathematical approaches listed includes explicitly the physical structure of the brain parenchyma at a microscopic level as an integral part of the model. Swanson et al. [20] consider the white matter fibres as an isotropic structure merely at a macroscopic level.

In this study, we will use a lattice-gas cellular automaton (LGCA) to demonstrate that the white matter fibres of the brain (prepattern) do influence the local and global growth pattern of the tumour indeed.

\section{Model}

We model the tumour system at a microscopic scale in which single brain and tumour cells are the basic entities. We assume that the growth of a vascularized GBM is mainly guided by the brain structure. The influence by the vascularization process is negligible. Furthermore, no diffusible substance is modeled, due to the same reason. Only tumour and brain cells were considered and only local interactions are taken into account. We do not include an explicit term for nutritive vascularization. Instead, we look at vessels as part of the morphological prepattern.

We describe the process of tumour cell invasion into normal brain tissue by using a cellular automaton (see figure 2, [31], for CA models). In our article, we use the term "cellular automata" in the sense of an infinite regular grid embedded in space. Each grid node has a state and a neighbourhood consisting of grid nodes. These neighbourhoods are translation invariant. Furthermore, state space and time are discrete. Within each time step the new states of the grid nodes will be simultaneously calculated. Therefore, only information given by the state in the neighbourhood and random noise will be considered. If models include moving cells, a special CA, a lattice gas cellular automaton (LGCA) has proved to be very useful. In a LGCA, the cells move from grid node to grid node and a reorientation takes place at each grid node.

Our automaton is a combination of a lattice-gas cellular automaton that describes the mobile tumour cells, and a simple cellular automaton for normal brain tissue. We distinguish three kinds of nodes: cancer, brain and "undefined" nodes. Starting with state $\mathrm{ca}_{t}$ it at time $t$, we calculate the state $\mathrm{ca}_{t+1}$ at time $t+1$ in a two-step process. In the first step, a new state $\mathrm{ca}_{t}{ }^{*}$ is chosen at each node simultaneously by a random process. The parameters for the random processes at each node are defined by the neighbourhood configuration. A second step of translocation simulates the moving behaviour of tumour cells. For computational reasons, we use a twodimensional square lattice embedded in the surface of an infinite cylinder; one dimension of which is periodic and the other (potentially) unlimited. Also death and birth of tumour cells and death of brain cells are simulated. Because we consider the Go or Grow hypothesis [32], 


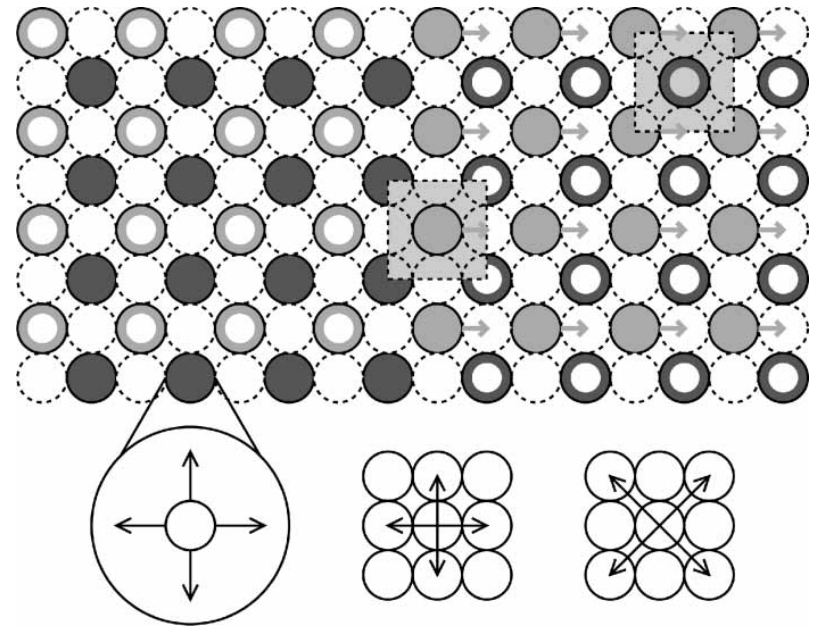

Figure 2. Cellular automaton for modeling cancer cell invasion. Top: Cancer nodes are dark grey and brain nodes are light grey. The diagram shows the initial state: the left area contains only cancer cells, the right area only brain cells (ring shape indicates empty node). The prepattern is shown as small gray arrows. The area of interaction controlled by density is shown as gray squares. Bottom, from left to right: channels of a cancer node (four non-zero-velocity channels and one zero-velocity channel), von Neumann neighborhood, cross neighborhood.

only resting tumour cells have a non-zero probability to proliferate.

\subsection{Cellular automaton and states}

The state of a brain node specifies the number of cells in the normal brain tissue. All cancer nodes together form a LGCA. The state $\mathbf{s}$ of a LGCA-node is defined as a configuration of particles (cancer cells) over channels. Each channel has a capacity, the maximal number of particles in this channel. For our LGCA, the set of all possible states is defined as

$$
\mathbf{L}=\mathbb{N} \times\{0,1\}^{4} \subset \mathbb{N}^{\{0, \ldots, 4\}},
$$

$(\mathbb{N}$ includes 0 ). We have one zero velocity or resting channel $\left(\mathbf{s}_{0}\right)$ with a potentially unlimited capacity and four non-zero velocity channels $\left(\mathbf{s}_{1}, \ldots, \mathbf{s}_{4}\right)$ with capacity one. Each non-zero velocity channel is associated with a direction of the von Neumann neighbourhood $\mathbf{N}$.

$$
\begin{aligned}
& \mathbf{N}=\left\{\mathbf{N}_{1}=(1,0), \mathbf{N}_{2}=(0,1),\right. \\
& \left.\mathbf{N}_{3}=(-1,0), \mathbf{N}_{4}=(0,-1)\right\} \\
& \mathbf{X}=\left\{\mathbf{X}_{1}=(1,1), \mathbf{X}_{2}=(-1,1),\right. \\
& \left.\mathbf{X}_{3}=(-1,-1), \mathbf{X}_{4}=(1,-1)\right\}
\end{aligned}
$$

The second neighbourhood $\mathbf{X}$ (cross neighbourhood) is the connection between brain and cancer CA nodes (see figure 2).
If $\mathbf{s} \in \mathbf{L}$ is a state of a cancer node, then the total number $|\mathbf{s}|$ of tumour cells is:

$$
|\mathbf{s}|=\sum_{k=0}^{4} \mathbf{s}_{k}
$$

and the local flux is

$$
\sum_{n=1}^{4} \mathbf{s}_{n} \mathbf{N}_{n}
$$

A state of the cellular automaton is a partial function

$$
\text { ca }: \mathbb{Z} \times \mathbb{Z}_{2 n} \rightarrow \mathbb{N} \cup \mathbf{L}
$$

which fulfills the following conditions:

$$
\mathrm{ca}(x, y) \in \mathbb{N} \Leftrightarrow(x, y) \in(2 \mathbb{Z}) \times\left(2 \mathbb{Z}_{2 n}\right)
$$

$$
\operatorname{ca}(x, y) \in \mathbf{L} \Leftrightarrow(x, y) \in(2 \mathbb{Z}+1) \times\left(2 \mathbb{Z}_{2 n}+1\right),
$$

where $2 n \in \mathbb{N}$ is the circumference of the cylinder. Equation (7) describes the normal brain tissue and equation (8) the cancer LGCA. To simplify the notation, we use $\mathbf{d}_{b}$ for an arbitrary brain and $\mathbf{d}_{c}$ for an arbitrary cancer node. The nearest cancer node in direction $v \in \mathbf{N}$ of a cancer node $\mathbf{d}_{c}$ is $\mathbf{d}_{c}+2 v$. This holds for brain nodes too. Please notice that there are still undefined values. (In an extended model we will use these values to simulate the interaction of tumour cells with the extracellular matrix.) Further, we assume that outside a finite region we will only find resting tumour cells in the left (equation (9)) and brain cells in the right (equation (10)) section. The desired cell density is given by $d$. The possible values for brain nodes are $0, d \in \mathbb{N}$ and for cancer nodes $(0,0,0,0,0)$, $(d, 0,0,0,0) \in \mathbf{L}$ (empty and only resting cells, respectively).

$$
\begin{aligned}
& \exists x_{0} \in \mathbb{Z} \quad \forall(x, y) \in \mathbb{Z} \times \mathbb{Z}_{2 n} \\
&\left(x<x_{0}\right) \Rightarrow \mathrm{ca}(x, y) \in\{0,(d, 0,0,0,0)\} \\
& \exists x_{1} \in \mathbb{Z} \quad \forall(x, y) \in \mathbb{Z} \times \mathbb{Z}_{2 n} \\
&\left(x_{1}<x\right) \Rightarrow \mathrm{ca}(x, y) \in\{d,(0,0,0,0,0)\}
\end{aligned}
$$

The set $\mathrm{CA}_{2 n}$ solely includes all possible states satisfying all conditions (7)-(9). 


\subsection{Target density of resting tumour cells}

For each brain and cancer node the local brain and cancer densities of cells $\left(\rho_{b}, \rho_{c}\right)$ are calculated as

$$
\begin{gathered}
\rho_{c}\left(\mathrm{ca}, \mathbf{d}_{c}\right)=\left|\mathrm{ca}\left(\mathbf{d}_{c}\right)\right| \\
\rho_{c}\left(\mathrm{ca}, \mathbf{d}_{b}\right)=\frac{1}{4} \sum_{n=1}^{4} \mathrm{ca}\left(\mathbf{H} \mathbf{d}_{b}+\mathbf{X}_{n}\right) \\
\rho_{b}\left(\mathrm{ca}, \mathbf{d}_{b}\right)=\mathrm{ca}\left(\mathbf{d}_{b}\right) \\
\rho_{b}\left(\mathrm{ca}, \mathbf{d}_{c}\right)=\frac{1}{4} \sum_{n=1}^{4} \mathrm{ca}\left(\mathbf{H} \mathbf{d}_{c}+\mathbf{X}_{n}\right) .
\end{gathered}
$$

We postulate that inter-cellular adhesion takes place between tumour cells and that its strength is a function of the local density of tumour cells. If the adhesion is high then tumour cells tend to rest. As a rough approximation for the complex adhesion process, we define the target density of resting tumour cells as the mean density of tumour cells in the neighbourhood.

$$
\rho_{r}\left(\mathrm{ca}, \mathbf{d}_{c}\right)=\frac{1}{4} \sum_{n=1}^{4} \rho_{c}\left(\mathrm{ca}, \mathbf{d}_{c}+2 \mathbf{N}_{n}\right)
$$

Due to the Go or Grow hypothesis, the (local) tumour growth is affected by this target density of resting tumour cells (see below).

\subsection{Target flux of tumour cells}

Non-resting cells are mobile and their movement is influenced by the densities of brain and tumour cells in the neighbourhood as well as by a prepattern. To find a new channel occupancy we have to define the net target flux $\mathbf{g}_{f}$ of tumour cells at each cancer node. This flux is a linear weighted combination of density gradients and the vector field of the prepattern. As a first step, we use a simple prepattern $\mathbf{g}_{p}$ generated by the brain cells.

$$
\begin{aligned}
\mathbf{g}_{f}\left(\mathrm{ca}, \mathbf{d}_{c}\right)= & \gamma_{p} \mathbf{g}_{p}\left(\mathrm{ca}, \mathbf{d}_{c}\right)+\gamma_{b} \mathbf{g}_{b}\left(\mathrm{ca}, \mathbf{d}_{c}\right) \\
& -\gamma_{c} \mathbf{g}_{c}\left(\mathrm{ca}, \mathbf{d}_{c}\right) \\
\mathbf{g}_{c}\left(\mathrm{ca}, \mathbf{d}_{c}\right)= & \sum_{n=1}^{4}\left|\mathrm{ca}\left(\mathbf{d}_{c}+2 \mathbf{N}_{n}\right)\right| \mathbf{N}_{n} \\
\mathbf{g}_{b}\left(\mathrm{ca}, \mathbf{d}_{c}\right)= & \sum_{n=1}^{4} \mathrm{ca}\left(\mathbf{d}_{c}+\mathbf{X}_{n}\right) \mathbf{X}_{n} \\
\mathbf{g}_{p}\left(\mathrm{ca}, \mathbf{d}_{c}\right)= & \frac{\rho_{b}\left(\mathbf{d}_{c}\right)}{\rho_{b}\left(\mathrm{ca}, \mathbf{d}_{c}\right)+\rho_{c}\left(\mathrm{ca}, \mathbf{d}_{c}\right)}(1,0)
\end{aligned}
$$

With equation (19), we incorporate a persistence in the movement of the malignant cells in our model which has been observed in in vitro studies [33]. The directed motion along the fibres is a presumed property of malignant cells and we analyse the effects of this on the global growth process. We are not interested in modeling the mechanism that is responsible for this persistence.

\subsection{Energy and probability}

For any given target density of resting cells and a given target flux of tumour cells, every possible local channel configuration of tumour cells is associated with an energy.

$$
\begin{aligned}
E\left(\mathbf{s}, \mathrm{ca}, \mathbf{d}_{c}\right)= & \Gamma_{r}\left(\rho_{r}\left(\mathrm{ca}, \mathbf{d}_{c}\right)-\mathbf{s}_{0}\right)^{2} \\
& +\Gamma_{f}\left\|\boldsymbol{g}_{f}\left(\mathrm{ca}, \mathbf{d}_{c}\right)-\sum_{n=1}^{4} \mathbf{s}_{n} \mathbf{N}_{n}\right\|^{2}
\end{aligned}
$$

The energy function $E$ measures the degree of fulfillment of the two properties: target density of resting cells and target flux of tumour cells. The two coefficients $\Gamma_{r}$ and $\Gamma_{f}$ determine the fraction of energy of these properties, respectively. The lower the fraction $\Gamma_{r} / \Gamma_{f}$ the more the energy depends on the flux of tumour cells. We use Boltzmann weights to choose one of all possible states at one node.

$$
\mathbf{s} \mapsto \exp \left(-E\left(\mathbf{s}, \mathrm{ca}, \mathbf{d}_{c}\right)\right)
$$

These weights define probabilities and one state is selected at random. This is performed for all cancer nodes $\mathbf{d}_{c}$ simultaneously to produce a new channel configuration $\mathrm{ca}_{t}^{\circ}\left(\mathbf{d}_{c}\right)$ for these nodes.

$$
\begin{aligned}
p\left(\mathbf{s}, \mathrm{ca}, \mathbf{d}_{c}\right) & = \begin{cases}\frac{\exp \left(-E\left(\mathbf{s}, \mathrm{ca}, \mathbf{d}_{c}\right)\right)}{\sum_{\left|\mathbf{s}^{\prime}\right|=|\mathbf{s}|} \exp \left(-E\left(\mathbf{s}^{\prime}, \mathrm{ca}, \mathbf{d}_{c}\right)\right)} & \text { if }|\mathbf{s}|=\left|\mathrm{ca}_{t}\left(\mathbf{d}_{c}\right)\right| \\
0 & \text { otherwise }\end{cases} \\
\mathrm{ca}_{t}^{\circ}\left(\mathbf{d}_{c}\right) & =\mathbf{s} \text { with probability } p\left(\mathbf{s}, \mathrm{ca}_{\mathrm{t}}, \mathbf{d}_{c}\right)
\end{aligned}
$$

This intermediate step considers only the reorientation of cancer cells. Before cancer cells move to their new positions proliferation (of cancer cells) and death (cancer and brain cells) take place.

\subsection{Birth and death}

Brain cell death, e.g. due to nutrition competition or induced apoptosis, is modeled as a function of local tumour cell density.

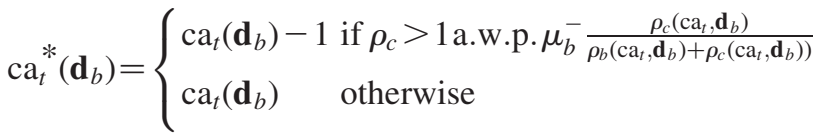

Here, we also have a random process: a.w.p. means "and with probability". The sensitivity of the process is 
controlled by the parameter $\boldsymbol{\mu}_{b}^{-}$. Birth and death of tumour cells are modeled as functions of local density represented by $\Phi^{+}$and $\Phi^{-}$, respectively. Only resting cells take part in these processes (Go or Grow hypothesis). A resting tumour cell proliferates at rate $\mu_{c}^{+}$only if space is available, whereas a resting tumour cell disappears at rate $\mu_{c}^{-}$if the local density is too high. Each random process is implemented as iteration over resting cells (variable $k$ in equations (27) and (28)).

$$
\mathrm{ca}_{t}^{*}\left(\mathbf{d}_{c}\right)=\Phi^{-}(\mathbf{s}_{0}^{+}, \mathbf{s}^{+}, \underbrace{\rho_{b}\left(\mathrm{ca}_{t}^{*}, \mathbf{d}_{c}\right)}_{\text {independent of cancer nodes }})
$$

with

$$
\begin{gathered}
\mathbf{s}^{+}=\Phi^{+}\left(\mathbf{s}_{0}^{\circ}, \mathbf{s}^{\circ}, \rho_{b}\left(\mathrm{ca}_{t}^{*}, \mathbf{d}_{c}\right)\right) \text { and } \mathbf{s}^{\circ}=\mathrm{ca}_{\mathrm{t}}^{\circ}\left(\mathbf{d}_{c}\right) \\
\Phi^{+}(k, \mathbf{s}, \rho)= \begin{cases}\mathbf{s} & \text { if }|\mathbf{s}|+\rho \geq d \text { or } k=0 \\
\Phi^{+}(k-1, \mathbf{s}+\mathbf{r}, \rho) & \text { if }|\mathbf{s}|+\rho \geq d \text { a.w.p. } \mu_{c}^{+} \\
\Phi^{+}(k-1, \mathbf{s}, \rho) & \text { otherwise }\end{cases} \\
\Phi^{-}(k, \mathbf{s}, \rho)= \begin{cases}\mathbf{s} & \text { if }|\mathbf{s}|+\rho \leq d \text { or } k=0 \\
\Phi^{-}(k-1, \mathbf{s}-\mathbf{r}, \rho) & \text { if }|\mathbf{s}|+\rho>d \text { a.w.p. } \mu_{c}^{-} \\
\Phi^{-}(k-1, \mathbf{s}, \rho) & \text { otherwise }\end{cases}
\end{gathered}
$$

$$
\text { with } \mathbf{r}=(1,0, \ldots, 0)
$$

\subsection{Translocation}

The translocation of the tumour cells is deterministic

$$
\forall n \in\{0, \ldots, 4\}: \mathrm{ca}_{t+1}\left(\mathbf{d}_{c}+2 \mathbf{N}_{n}\right)_{n}=\mathrm{ca}_{t}^{*}\left(\mathbf{d}_{c}\right)_{n},
$$

with $N_{0}=(0,0)$ and ca $\ldots(\ldots)_{n}$ is the channel in direction $n$. As there is no translocation of brain cells,

$$
\mathrm{ca}_{t+1}\left(\mathbf{d}_{b}\right)=\mathrm{ca}_{t}^{*}\left(\mathbf{d}_{b}\right) \text {. }
$$

\section{Simulations}

We implemented the above model as a $\mathrm{C}++$ program and used a pseudo random generator to simulate the probabilities. The program generates chains of configurations for the whole cellular automaton:

$$
\mathrm{ca}_{0} \rightarrow \mathrm{ca}_{1} \rightarrow \mathrm{ca}_{2} \rightarrow \cdots \rightarrow \mathrm{ca}_{t-1} \rightarrow \mathrm{ca}_{t} \rightarrow \mathrm{ca}_{t+1} \rightarrow \cdots
$$

Such a chain is a realisation of the Markov-like two-step process. The outcome of the simulation is controlled by ten parameters:

$\begin{array}{ll}\text { General } & \\ 2 n & \text { circumference of cylinder } \\ d & \text { desired density } \\ \text { Gradient } & \\ \gamma_{p} & \text { prepattern } \\ \gamma_{b} & \text { brain cells } \\ \gamma_{c} & \text { tumour cells } \\ \text { Tumour } & \\ \Gamma_{r} & \text { rest } \\ \Gamma_{f} & \text { flux } \\ \mu_{c}^{+} & \text {birth } \\ \mu_{c}^{-} & \text {death } \\ \text { Brain } & \\ \mu_{b}^{-} & \text {death }\end{array}$

For all simulations, we set $2 n=50$ and $d=4$. The initial state $\mathrm{ca}_{0}$ is (see also figure 2):

$$
\mathrm{ca}_{0}\left(\mathbf{d}_{c}\right)= \begin{cases}(d, 0, \ldots, 0) & \text { if } \mathbf{d}_{c}=(x, y) \text { and } x<10 \\ (0,0, \ldots, 0) & \text { otherwise }\end{cases}
$$

$$
\mathrm{ca}_{0}\left(\mathbf{d}_{b}\right)= \begin{cases}0 & \text { if } \mathbf{d}_{b}=(x, y) \text { and } x<10 \\ d & \text { otherwise }\end{cases}
$$

For computational simplification, the state $\mathrm{ca}_{t}(x, y)$ is kept constant for all $t$ if $x<2$. Please notice that the ratio of the total number of cancer and brain cells does not correspond to any biological or clinical property. We only simulate a small fraction of a real brain tumour.

We used a brute force method to scan the parameter space. For each parameter set, we calculate and analyse the mean density of malignant cells and brain cells along the $y$-axis as functions of the $x$-position and time. Out of the five resulting growth patterns $(\mathrm{I})-(\mathrm{V})$ that can be observed (see figure 3), we only consider the first three. In pattern (IV) the resting probability of the malignant cells is zero and only the prepattern decides on the movement of the malignant cells and in pattern (V) the constant states $\mathrm{ca}_{t}(x, y)$ at the left boundaries constitute the source of the cell flux. We disregard these two patterns as being side effects of the boundaries of the model. In addition to the results shown in figure 3, the observed local density of malignant cells in the cancer bulk can vary with the parameters (above or below the target density $d$ ).

The three travelling wave-like patterns (I)-(III) differ in the time evolution of their shape. In the first pattern (I), the shape is nearly constant in time and there are no isolated single invading cancer cells farther away from the cancer bulk. The second pattern (II) differs from the first one in that two growth speeds can be observed: the speed of the cancer bulk front and the higher speed of invasion of single malignant cells into the healthy brain tissue. The shape of the travelling wave splits into two 
(I)

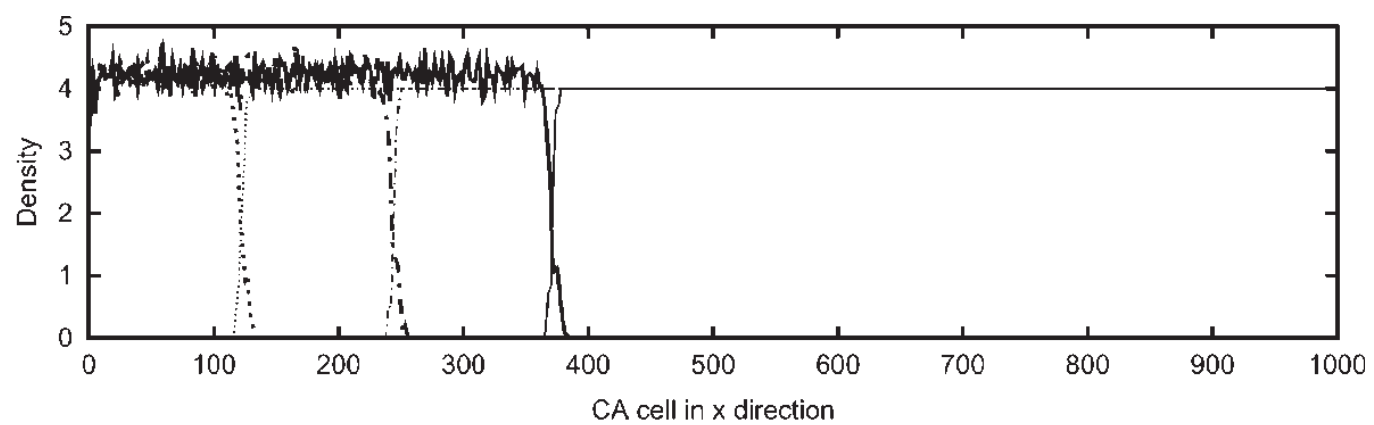

(II)
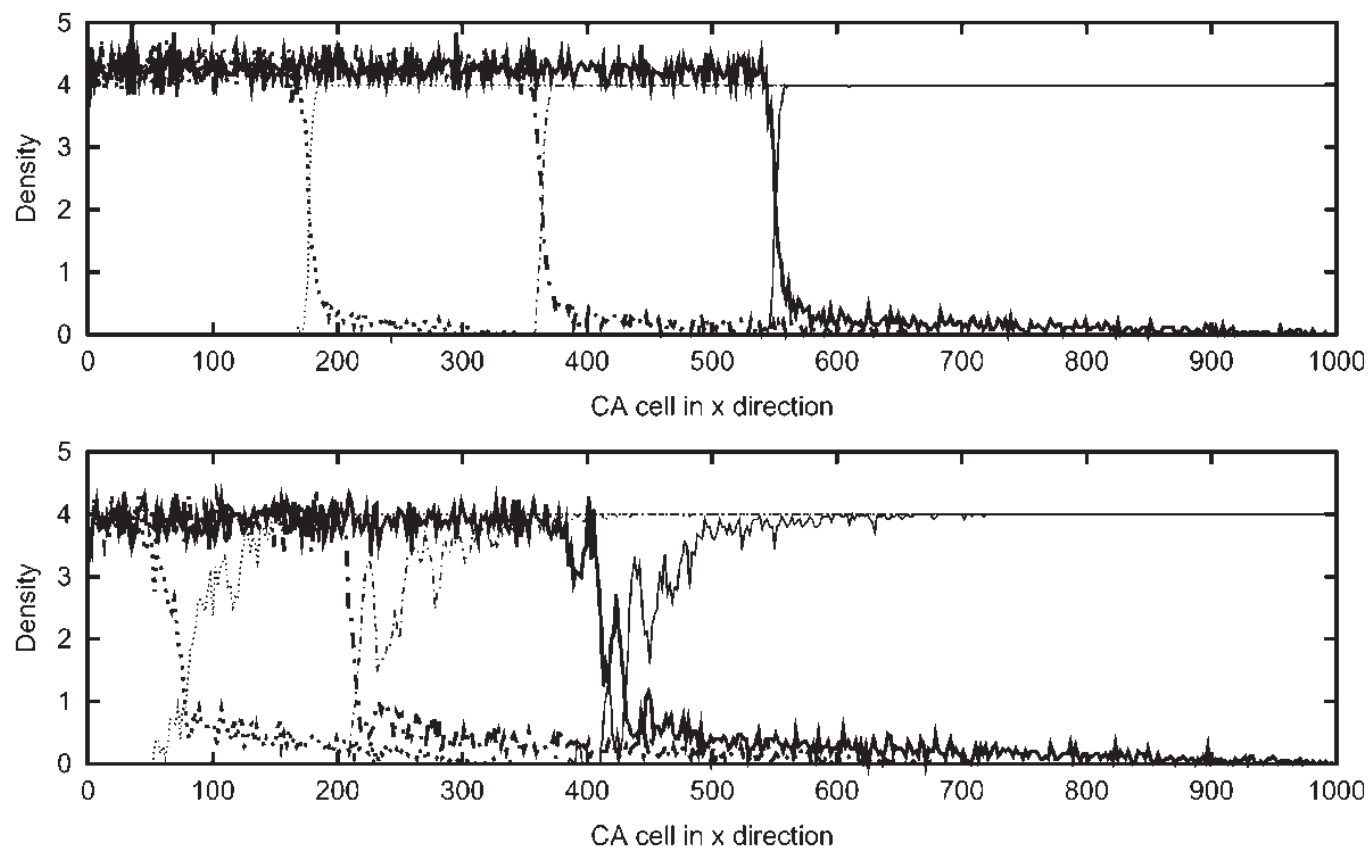

(IV)

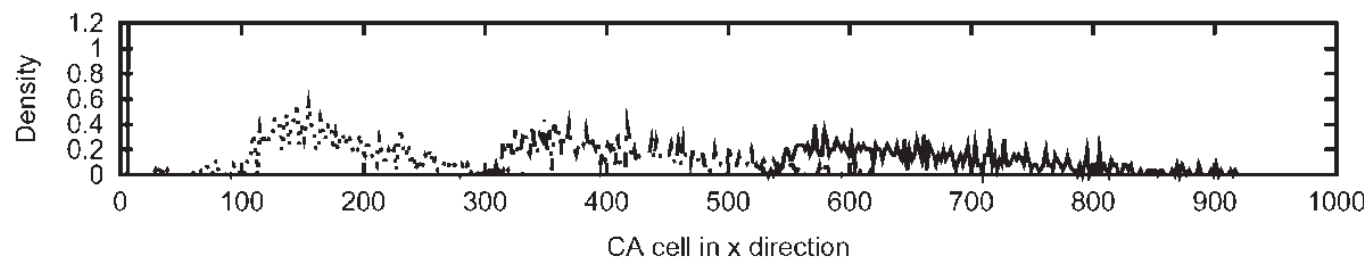

(V)

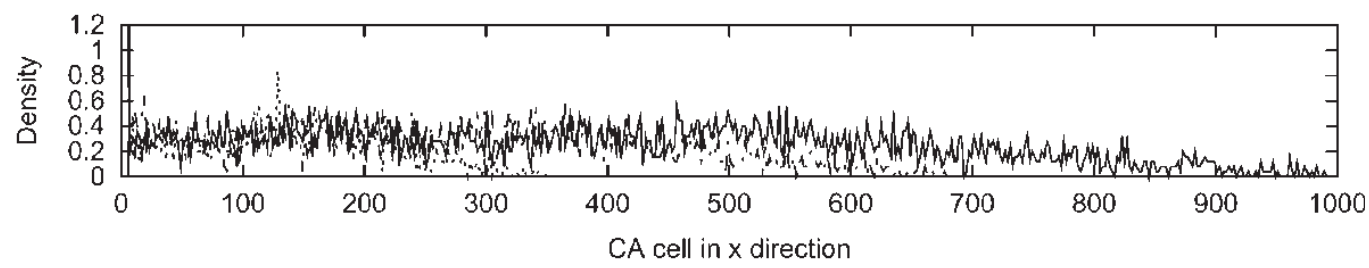

\begin{tabular}{lcccccccc} 
& $\mu_{b}^{-}$ & $\mu_{c}^{-}$ & $\mu_{c}^{+}$ & $\gamma_{b}$ & $\gamma_{c}$ & $\gamma_{p}$ & $\Gamma_{r}$ & $\Gamma_{f}$ \\
\hline (I) & 1 & 1 & 1 & 1 & 0 & 0 & 1 & 1 \\
(II) & 1 & 1 & 1 & 0 & 1 & 1 & 1 & 1 \\
(III) & 1 & 0.1 & 0.1 & 0.1 & 0 & 1 & 1 & 1 \\
(IV) & 0.1 & 0.1 & 0.1 & 0 & 1 & 1 & 0 & 1 \\
(V) & 0.1 & 0.1 & 0.1 & 0.1 & 0 & 1 & 1 & 1
\end{tabular}

Figure 3. Observable growth behaviour. The mean densities (i.e. mean number of tumour cells (thick line) or brain cells (thin line) per CA node) in $x$ direction alter 300 (dotted line), 600 (dashed line) and 900 (solid line) time units are shown. The local density of malignant cells can vary with the parameters (not shown). Traveling wave-like behavior: (I): Constant shape in time, no invading cells far away from the cancer bulk. (II): Bulk boundary with constant speed and invading cells with higher speed. (III): Like (II) but with density instabilities. Artefacts: Caused by boundary conditions and the approximation of the prepattern as a vector field. The density of brain cells is nearly constant over time. (IV): Detached traveling wave and only invading cells without a cancer bulk. (V): Constant cell flux caused by boundary condition at the left edge of the LGCA. 
(A)

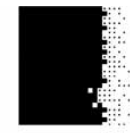

(B)

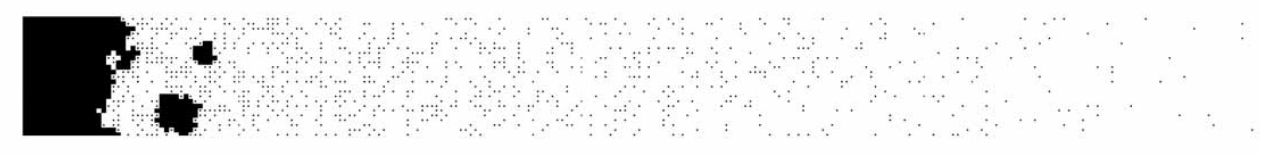

(C)

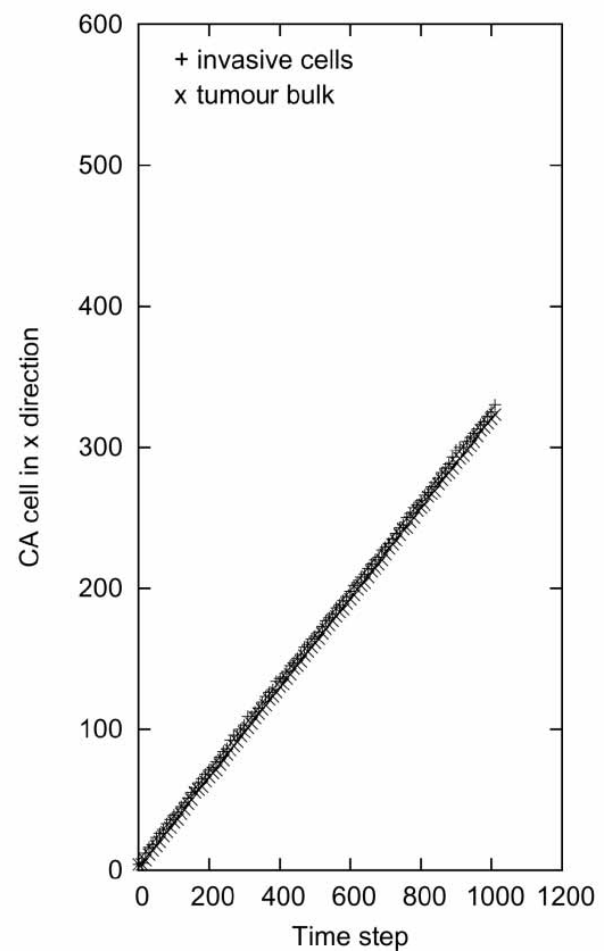

(D)

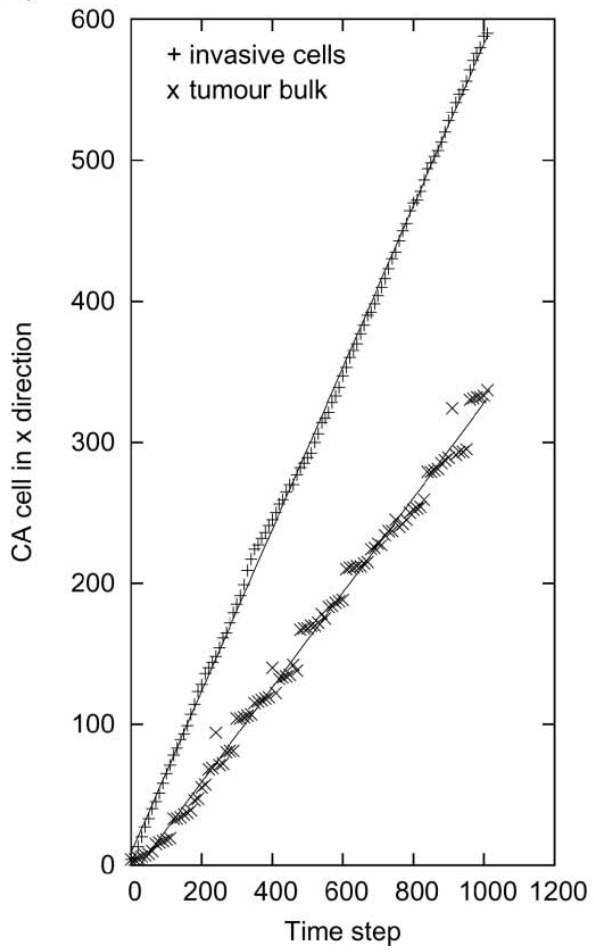

Figure 4. Time evolution of two characteristic invasion behaviours (A) + (B): Tumour growth from left to right: malignant cells are visualised as black dots; one can distinguish tumour bulk and single invading cells. (A): Without influence of prepattern. The observed growth pattern corresponds to a benign cancer in the grey matter with expansive growth and (almost) no invasion. (B): With influence of prepattern. The growth pattern corresponds to a malignant tumour in the white matter. Due to the high density of malignant cells in front of the cancer bulk cancer islets are observable. (C) + (D): Position of the rightmost CA node marked as tumour bulk and the rightmost malignant cells (see top row) in time. The invasion speed can be calculated as the slope of the linear regression line. (C): No influence of the prepattern. The speeds of invasion front and of tumour boundary do not differ. (D): Due to the influence of the prepattern the tumour boundary changes. Now the speed of the invading front is higher then the speed of the tumour boundary (corresponding to the situation shown in the top row).

parts: the section dependent on the bulk boundary is constant in time and only the density section of the moving cells grows in length. This occurs only if $\gamma_{p} \Gamma_{f}$ is greater than zero. In the third pattern (III) density instabilities can additionally be observed. A time series of the two-dimensional density plots of pattern (III) identifies growing islets of malignant cells just in front of the tumour bulk (see figure 4). Due to the existence of these islets, the tumour front develops in a discontinuous fashion (see (D) in figure 4).

To compare the observed speeds we use the slope of a linear approximation of the growth process. We define the CA node as part of the cancer bulk if the local tumour density is at least the same as local brain density $\left(\rho_{c} \geq \rho_{b}\right)$. The speed of the bulk boundary is calculated by linear regression of the $x$-coordinate of the rightmost cancer bulk CA node position, which depends on time (see figure 4)

$$
\begin{aligned}
\operatorname{pos}_{\text {bulk }}(t)= & \max \{x \mid \exists y \\
& \left.: \operatorname{ca}_{t}(x, y) \text { is part of the cancer bulk }\right\} .
\end{aligned}
$$

The speed of single invading cells is calculated by linear regression of the $x$-coordinate of the rightmost malignant cell position, which depends on time

$$
\begin{aligned}
\operatorname{pos}_{\text {single }}(t)= & \max \left\{x \mid \exists y: \mathrm{ca}_{t}(x, y)\right. \text { is cancer node } \\
& \text { and } \left.\left|\mathrm{ca}_{t}(x, y)\right|>0\right\}
\end{aligned}
$$

The speed $v_{\text {bulk }}$ of the boundary of the cancer bulk and the speed $v_{\text {single }}$ of single invading cells are equal 
in pattern (I) and unequal in patterns (II) and (III). We inspect the fraction $v_{\text {bulk }} / v_{\text {single }}$ because $v_{\text {bulk }} \leq$ $v_{\text {single }}$. If $v_{\text {bulk }} / v_{\text {single }}=1$, the speed does not differ and if $v_{\text {bulk }} / v_{\text {single }}<1$ the invading front is faster then the bulk boundary. In order to eliminate random side effects we repeated the simulations 10 times for each parameter set and calculated the mean value and second moment of the speeds. For each parameter set, the calculated speeds have only a small standard deviation. Typical results are shown in figure 5 .

The invasion speed $v_{\text {single }}$ highly depends on the product $\gamma_{p} \Gamma_{f}$ controlling the prepattern (see figure 5 , left column). The bulk speed is, for example, controlled by the death rate $\mu_{b}^{-}$of brain cells. If $\mu_{b}^{-}$is large, the proliferation of malignant cells is not restricted by space due to the higher probability for brain cells to be killed. And if there is a high flux of malignant cells $\left(\gamma_{p} \Gamma_{f}\right.$ is high), then there is more space for proliferation and the bulk speed increases. The ratio $v_{\text {bulk }} / v_{\text {single }}$ is 1 if $\gamma_{p} \Gamma_{f}=0$ but if $\gamma_{p} \Gamma_{f}>0$ we observe a different speed ratio and the ratio $v_{\text {bulk }} / v_{\text {single }}$ is less than one. With growing $\gamma_{p} \Gamma_{f}$, the ratio $v_{\text {bulk }} / v_{\text {single }}$ decreases nearly linearly, but if the influence of the prepattern reaches a parameter-dependent level we observe a saturation in the ratio $v_{\text {bulk }} / v_{\text {single }}$ (see figure 5 ).

\section{Discussion}

We have introduced a lattice-gas cellular automaton to simulate and to analyse the influence of the fibre tract structure of the brain on the development of the brain tumour Glioblastoma multiforme. In the model, we consider healthy immobile brain tissue and potentially mobile malignant cells. Malignant cells may move or rest and only resting malignant cells proliferate (Go or Growth hypothesis). Movement and resting behaviour are controlled by local cell densities and density gradients of brain and cancer cells (haptotaxis). Chemotaxis and any cellular interactions not dependent on cell densities are not included in the model. The brain tissue is destroyed by cancer cells and it is assumed that the brain can not regenerate. The physical structure of the brain, in particular its white matter tracts are considered as a prepattern represented by a vector field, which introduces unidirectional persistence in malignant cell movement. Such persistence of GBM cell movement has been experimentally observed.

We have characterised the speeds of bulk boundary and malignant cell invasion and found three different travelling wave-like growth patterns (figure 3 (I)-(III)). In the first scenario (I), a constant travelling wave shape without invading cells far away from the cancer bulk is observed. The travelling wave shape is non-constant in the second scenario (II) because here invading cells possess a higher speed than the bulk boundary. The third scenario (III) is similar to the second, but, additionally, density instabilities appear, visible as growing islets of malignant cells just in front of the tumour bulk (see also figure 4). In addition, there are artefact scenarios with only invading cells and without a growing cancer bulk. Particularly, in scenario (IV) there are no resting cells $\left(\Gamma_{r}=0\right)$ and thus there is no proliferation; due to the imposed prepattern a moving cluster of malignant cells emerges which is composed of the cells of our initial state. In scenario (V) proliferation $\left(\Gamma_{r}>0\right)$ occurs, but almost only at the left boundary due to the boundary condition (there are always malignant cells at the left boundary). In both cases the brain tissue remains nearly unchanged because the death rate of brain cells $\mu_{b}^{-}$and the density of malignant cells are small.

The main difference between scenario (I) and scenarios (II) and (III) is the absence and presence of the prepattern, respectively. Even under a small influence of the prepattern $\left(\gamma_{p} \Gamma_{f}>0\right)$, isolated malignant cells can be detected far away from the cancer bulk. Note that no density gradients of brain or cancer cells are required to observe this behaviour. Without the influence of the "fibrous" prepattern isolated cells are only found in the vicinity of the bulk.

\subsection{Scenario (I)}

The observed growth pattern in scenario (I) corresponds to a "benign" cancer in the grey matter with expansive growth and (almost) no invasion. Clinically, such a growth mode is visible within the grey matter. This behaviour is observed only if there is no influence of the "fibrous" prepattern $\left(\gamma_{p} \Gamma_{f}=0\right)$ and is nearly independent of the other parameters. In addition, surrounding tissue is dissolved by direct contact of cancer bulk and brain tissue. Scenario (I) is comparable to travelling wave solutions in the reaction-advection model analysed in Perumpanani et al. [14] and Marchant et al. [15,16]. Here, as in our lattice-gas cellular automaton only malignant cells and surrounding tissue cells are considered. Additionally, in the cited model the tissue is dissolved by a non-diffusible protease secreted by the tumour cells. Furthermore, in order to simulate proliferation of tumour cells the authors use a bounded non-linear term (as in the Fisher-KPP equation, [34,35]) and the movement along the density gradient of the tissue (haptotaxis) has been explicitly taken into account. In a reduced 1D-PDE system a travelling wave solution with unlimited support (i.e. the density function of malignant cells is non-zero everywhere) is observed, which declines quickly towards zero, and, in addition, shock wave-like solutions (unlimited only in one direction and declining rapidly to zero in the other direction). In contrast, in our model without influence of the prepattern $\left(\gamma_{p} \Gamma_{f}=0\right)$ we also find a rapid decay of tumour cells in the observed travelling wave but no shock wave-like pattern. The explanation for the rapid decay is the random walk of isolated tumour cells in the homogeneous healthy brain tissue. This undirected movement limits the speed $\left(v_{\text {single }}\right)$ and range of the invasion front. In combination with the proliferation of tumour cells and the death of brain cells, the random walk 

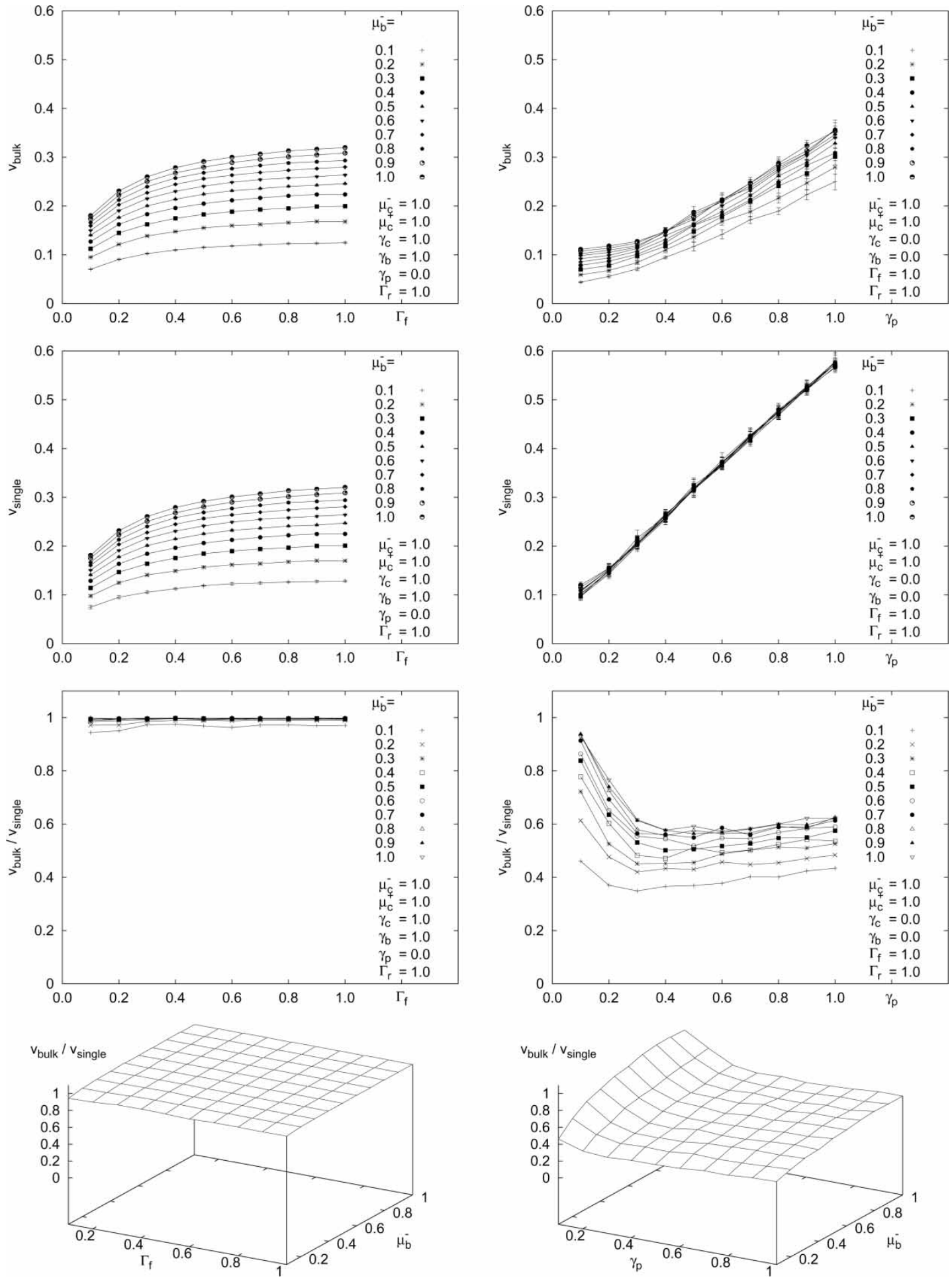

Figure 5. Typically observed speeds of bulk boundary and invading front. Left: Scenario (I), without influence of the prepattern. The tumour bulk growth $\left(v_{\text {bulk }}\right)$ is nearly as fast as the invasion front $\left(v_{\text {single }}\right)$ due to the random walk of malignant cells. This undirected movement also reduces the expansion space in front of the tumour and therefore proliferation is limited. The death rate of the brain cells determines the maximal speed of tumour expansion. Right: Scenario (II), with influence of prepattern only. Due to the directed random walk, the invasion front is slowed down only slightly. The tumour bulk growth speed is increased because the malignant cell flux towards the brain tissue creates a constantly available expansion space just in front of the tumour bulk which allows further proliferation. This is also the reason why the maximum speed does not approach any limit. The speed of the tumour bulk is lower than the speed of the invasion front due to the death rate of brain cells. Except for a short transition phase the ratio of both speeds is constant.

produces a bulk speed $v_{\text {bulk }}$ as fast as $v_{\text {single. }}$ Because a travelling wave analysis of a CA system is much more complicated than of a PDE system we diagrammed only numerical results in figure 5 .
The speeds of cell movement converge to a maximum (see figure 4) with increasing influence of density gradients $\left(\Gamma_{f} \uparrow 1\right)$. This can be explained as follows: because the more malignant cells lose contact to 
the tumour bulk, the more expansion space for the tumour growth exists in front of the tumour bulk and due to the proliferation process this space is filled up with new malignant cells. The speed $v_{\text {bulk }}$ of the bulk is then limited by the death rate of the brain cells (the higher this rate the higher the speed).

\subsection{Scenario $(I I+I I I)$}

The growth pattern in scenario (II) and (III) corresponds to a malignant tumour in the white matter. The pattern is mainly caused by the prepattern. Even if the influence of the fibre structure is very small $\left(\gamma_{p} \Gamma_{f}>0\right)$, we observe a flux of malignant cells moving ahead of the tumour into the healthy brain. The prepattern allows persistent cell movement even through homogeneous (uniform density) tissue regions. In particular, the existence of density gradients (as in chemo- or haptotaxis cell movement) is not required. In addition, $v_{\text {bulk }} / v_{\text {single }}<1$ (see figures 3 and 5), since, due to the directed random cell motion, the invasion front is slowed down only slightly, contrary to the tumour bulk that is slowed down significantly due to interaction with brain cells. This is again a travelling wave-like behaviour even if the travelling wave shape is not constant. No such behaviour has been described so far for PDE systems modeling tumour invasion. Note that movement of tumour cells that have left the tumour bulk remains directed since the "fibrous" prepattern induces a movement preference also in the homogeneous brain region. Because of the directed movement of malignant cells, there is less crowding and more space for expansion, i.e. proliferation. Accordingly, the number of tumour cells grows faster with than without a prepattern. Because the degradation probability of brain cells is a function of local cancer cell density, the tissue in the immediate vicinity of the tumour bulk is degraded with a larger probability due to the prepattern-imposed cell flow. The combination of enhanced proliferation of tumour cells and degradation of healthy brain tissue implies a higher growth speed of the tumour bulk compared to scenario (I). The same reasoning explains why no saturation is observed: after a short transitional phase in which random movement dominates the directed tumour cell movement (depending on the parameter weights) the ratio of both speeds $v_{\text {bulk }}$ and $v_{\text {single }}$ remains constant.

\subsection{Scenario (III)}

The surface of the bulk in scenario (III) loses its connectivity and normal brain tissue is incorporated into the tumour. The density in the flux of malignant cells is high and hence the probability for malignant cells to rest is high. In combination with proliferation (only possible for resting cells) we observe a density instability: the higher the flux of invading cells, the higher the density of malignant cells in front of the cancer bulk and the higher the probability for the creation of small "cancer islets" (see figure 3). The jumps in time evolution of the tumour front in figure 4 originate from these islets.

Note that in our lattice-gas cellular automaton we have not explicitly modeled subpopulations of cancer cells but distinguish moving and resting malignant cells. This is justified since from a biological point of view it is not clear how to subdivide the malignant cell population in disjunct subpopulations with different properties. In particular, we have not explicitly modeled any flux between subpopulations of malignant cells as, e.g. in Sherratt and Chaplain [17] or in the hybrid PDE/CA models of Dormann and Deutsch [28] and Anderson [30]. An approximating continuous PDE system for our LGCA model would be a single-species advection reaction-diffusion model, in which the parameters (e.g. diffusion coefficient or proliferation rate) are functions of the local cancer cell density.

In the linear reaction-diffusion model of Swanson (see e.g. [20]) the orientational information about fibrous brain structures is not considered. In this model, cell motion is approximated by isotropic diffusion, which corresponds to the assumption that the movement of single malignant cells is random even in regions of high coherency of fibre structures (as in the corpus collosum). A spatio-temporal analysis of the invasion front is only possible indirectly via the definition of detection thresholds. In addition, one can correlate the temporal evolution of tumour volume with the mean time of survival. Travelling wave solutions do not appear due to the linear formulation of the reactiondiffusion equation.

Although we have so far considered only a first approximation of the complex developmental process of a GBM in the proposed lattice-gas cellular automaton model, clinical observations agree in principle with the results of our model-based analysis. For instance, it is a common assumption that the origin of a butterfly-shaped GBM lies in one hemisphere, and then grows very fast through the corpus collosum so that a symmetrically shaped cancer is observable in both halves of the brain. The fast traverse through the corpus collosum is explained by the highly parallel aligned fibres in this anatomical structure. The aim of our further work is to collect these data from the clinical practice of neurosurgery and analyse the relation of fibre structure and speed of infiltration. In the next step, our model will be validated against original clinical and radiographic data.

\section{References}

[1] Kleihues, P., Burger, P.C. and Scheithauer, B.W. (Eds), 1993, Histological Typing of Tumours of the Central Nervous System (Springer: Berlin).

[2] Chicoine, M.R. and Silbergeld, D.L., 1995, Invading C6 glioma cells maintaining tumorigenicity. J. Neurosurg., 83(4), 665-671.

[3] Silbergeld, D.L. and Chicoine, M.R., 1997, Isolation and characterization of human malignant glioma cells from histologically normal brain. J. Neurosurg., 86(3), 525-531.

[4] Gaspar, L.E., Fisher, B.J., Macdonald, D.R., LeBer, D.V., Halperin E.C., Schold, Jr. S.C. and Cairncross, J.G., 1992, Supratentorial malignant glioma: patterns of recurrence and implications for 
external beam local treatment. Int. J. Radiat. Oncol. Biol. Phys., 24(1), 55-57.

[5] Yung, W., Albright, K.E., Olson, R.J., Fredericks, R., Fink, K., Prados, D., Brada, M.M., Spence, A., Hohl, J., Shapiro, R.W., Glantz, M., Greenberg, H., Selker, G., Vick, R.A., Rampling, N.R., Friedman, H., Phillips, P., Bruner, J., Yue, N., Osoba, D., Zaknoen, S. and Levin, A.V., 2000, A phase II study of temozolomide vs. procarbazine in patients with Glioblastoma multiforme at first relapse. Br. J. Cancer, 83(5), 588-593.

[6] Westphal, M., Hilt, C., Bortey, D.E., Delavault, P., Olivares, R., Warnke, C., Whittic, P.R., Jaaskelainen, I.J. and Ram, Z., 2003, A phase 3 trial of local chemotherapy with biodegradable carmustine (BCNU) wafers (Gliadel wafers) in patients with primary malignant glioma. Neuro-oncology, 5(2), 79-88.

[7] Davis, F., Freels, S., Grutsch, J., Barlas, S. and Brem, S., 1998, Survival rates in patients with primary malignant brain tumors stratified by patient age and tumor histological type: an analysis based on Surveillance, Epidemiology, and End Results (SEER) data, 1973-1991. J. Neurosurg., 88, 1-10.

[8] Scherer, H.J., 1940, The forms of growth in gliomas and their practical significance. Brain, 63, 1-35.

[9] Matsukado, Y., MacCarty, C.S. and Kemohan, J.W., 1961, The growth of Glioblastoma multiforme (astrocytomas, grades 3 and 4) in neurosurgical practice. J. Neurosurg., 18, 636-644.

[10] Giese, A. and Westphal, M., 1996, Glioma invasion in the central nervous system. Neurosurgery, 39(2), 235-250.

[11] Aboody, K.S., Brown, A., Rainov, N.G., Bower, K.A., Liu, S., Yang, W., Small, J.E., Herrlinger, U., Ourednik, V., Black, P.M., Breakefield, X.O. and Snyder, E.Y., 2000, Neural stem cells display extensive tropism for pathology in adult brain: evidence from intracranial gliomas. Proc. Natl Acad. Sci., 97(23), 12846-12851.

[12] Maher, E.A., Furnari, F.B., Bachoo, R.M., Rowitch, D.H., Louis, D.N., Cavenee, W.K. and DePinho, R.A., 2001, Malignant glioma: genetics and biology of a grave matter. Genes Dev., 15, 1311-1333.

[13] de Boüard, S., Christov, C., Guillamo, J.S., Kassar-Duchossoy, L., Palfi, S., Leguerinel, C., Masset, M., Cohen-Hagenauer, O., Peschanski, M. and Lefrancois, T., 2002, Invasion of human glioma biopsy specimens in cultures of rodent brain slices: a quantitative analysis. J. Neurosurg., 97(1), 169-176.

[14] Perumpanani, A.J., Sherratt, J.A., Norbury, J. and Byrne, H.M., 1999, A two parameter family of travelling waves with a singular barrier arising from the modelling of extracellular matrix mediated cellular invasion. Physica D, 126, 145-159.

[15] Marchant, B.P., Norbury, J. and Perumpanani, A.J., 2000, Travelling shock waves arising in a model of malignant invasion. SIAM J. Appl. Math., 60(2), 263-476.

[16] Marchant, B.P., Norbury, J. and Sherratt, J.A., 2001, Travelling wave solutions to a haptotaxis-dominated model of malignant invasion. Nonlinearity, 14, 1653-1671.

[17] Sherratt, J.A. and Chaplain, M.A.J., 2001, A new mathematical model for avascular tumour growth. J. Math. Biol., 43, 291-312.

[18] Tracqui, P., Cruywagen, G.C., Woodward, D.E., Bartoo, G.T., Murray, J.D. and Alvord, Jr. E.C., 1995, A mathematical model of glioma growth: the effect of chemotherapy on spatio-temporal growth. Cell Prolif., 28, 17-31.

[19] Woodward, D.E., Cook, J., Tracqui, P., Cruywagen, G.C., Murray, J.D. and Alvord, Jr. E.C., 1996, A mathematical model of glioma growth: the effect of extent of surgical resection. Cell Prolif., 29, 269-288.

[20] Swanson, K.R., Alvord, E.C. and Murray, J.D., 2000, A quantitative model for differential motility of gliomas in grey and white matter. Cell Prolif., 33(5), 317-330.

[21] Swanson, K.R., Alvord, Jr. E.C. and Murray, J.D., 2002, Quantifying efficacy of chemotherapy of brain tumors (gliomas) with homogeneous and heterogeneous drug delivery. Acta Biotheor, 50(4), 223-237.

[22] Swanson, K.R., Alvord, Jr. E.C. and Murray, J.D., 2002, Virtual brain tumors (gliomas) enhance the reality of medical imaging and highlight inadequacies of current therapy. Br. J. Cancer, 86, $14-18$.

[23] Swanson, K.R., Alvord, Jr. E.C. and Murray, J.D., 2003, Virtual resection of gliomas: effects of location and extent of resection on recurrence. Special Issue: Modeling and Simulation of Tumour Development, Treatment, and Control. Math. Comput. Modelling, 37, 1177-1190.

[24] Swanson, K.R., Bridge, C., Murray, J.D. and Alvord, Jr. E.C., 2003, Virtual and real brain tumors: using mathematical modeling to quantify glioma growth and invasion. J. Neural. Sci., 216(1), $1-10$.

[25] Moreira, J. and Deutsch, A., 2002, Cellular automaton models of tumor development: a critical review. Adv. Complex Syst., 5(2-3), 247-267.

[26] Patel, A.A., Gawlinski, E.T., Lemieux, S.K. and Gatenby, R.A., 2001, A cellular automaton model of early tumor growth and invasion: the effects of native tissue vascularity and increased anaerobic tumor metabolism. J. Theor. Biol., 213(3), 315-331.

[27] Sander, L.M. and Deisboeck, T.S., 2002 Growth patterns of microscopic and brain tumors, Available online at: http://arxiv.org/abs/physics/0206066.

[28] Dormann, S. and Deutsch, A., 2002, Modeling of self-organized avascular tumor growth with a hybrid cellular automaton. In Silico Biol., 2, 0035.

[29] Alarcón, T., Byrne, H. and Maini, P., 2003, A cellular automaton model for tumour growth in inhomogeneous environment. J. Theor. Biol., 225(2), 257-274.

[30] Anderson, A.R.A., 2004, Solid tumour invasion: the importance of cell adhesion. In: A. Deutsch, M. Falcke, J. Howard and W. Zimmermann (Eds.) Function and Regulation of Cellular Systems: Experiments and Models (Birkhäuser: Basel).

[31] Deutsch, A. and Dormann, S. 2004, Cellular Automaton Modeling of Biological Pattern Formation (Birkhäuser: Boston).

[32] Corcoran, M.S. and Del Maestro, M.D. Rolando F., 2003, Testing the 'GO OR GROW' hypothesis in human medulloblastoma cell lines in two and three dimensions. Neurosurgery, 53(1), 174-184.

[33] Demutu, T., Hopf, N.J., Kempski, O., Sauner, D., Herr, M., Giese, A. and Perneczky, A., 2001, Migratory activity of human glioma cell lines in vitro assessed by continuous single cell observation. Clin. Exp. Metastasis, 18, 589-597.

[34] Fisher, R.A., 1937, The wave of advance of advantageous genes. Ann. Eugenics, 7, 355-369.

[35] Kolmogorov, A., Petrovsky, A. and Piscounoff, N., 1988, Study of the diffusion equation with growth of the quantity of matter and its application to a biology problem. In: P. Pelce (Ed.) Dynamics of Curved Fronts (Boston: Academic Press). 


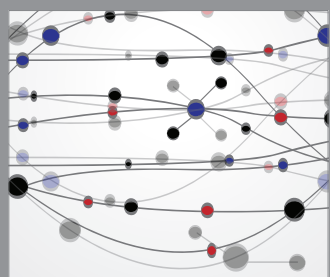

The Scientific World Journal
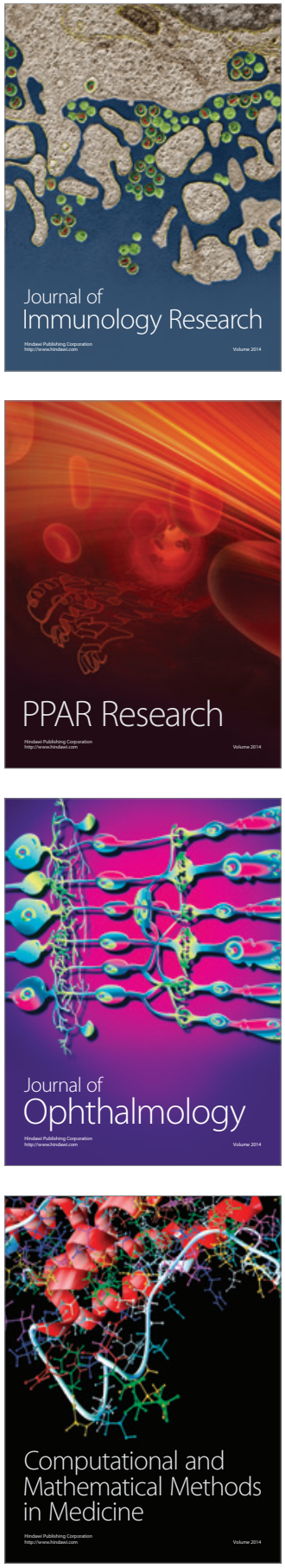

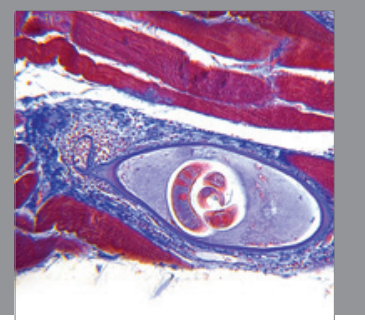

Gastroenterology

Research and Practice
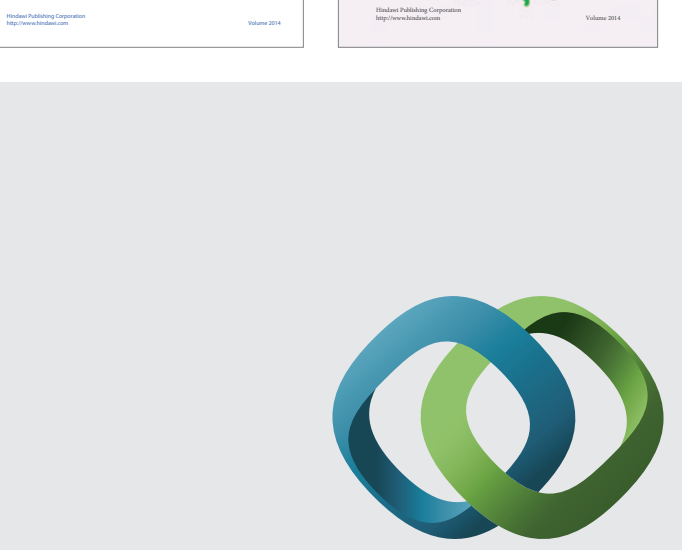

\section{Hindawi}

Submit your manuscripts at

http://www.hindawi.com
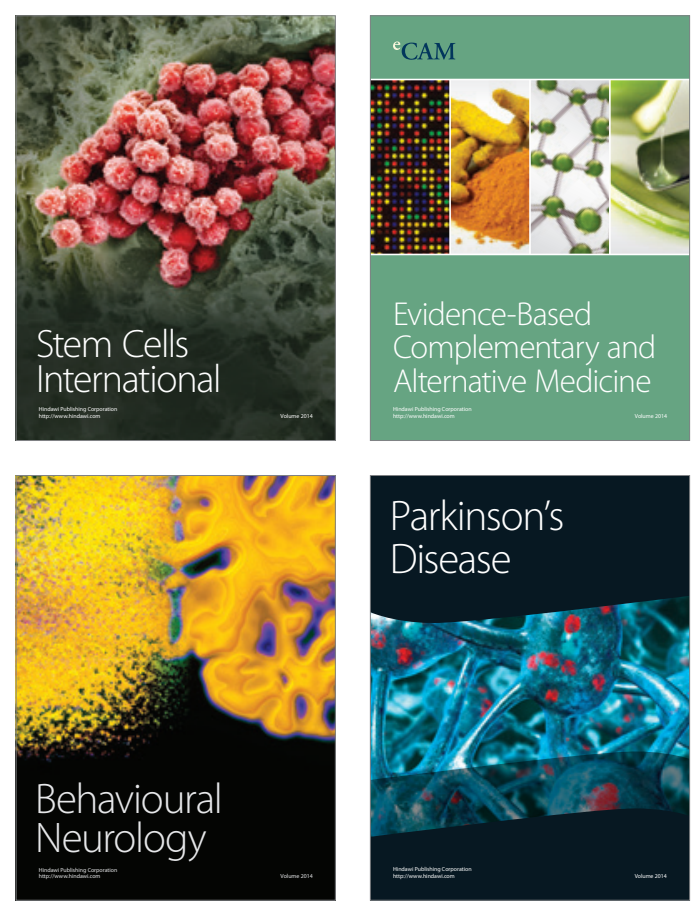

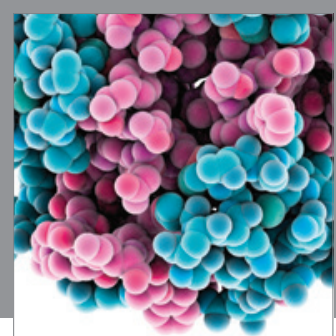

Journal of
Diabetes Research

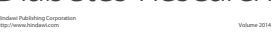

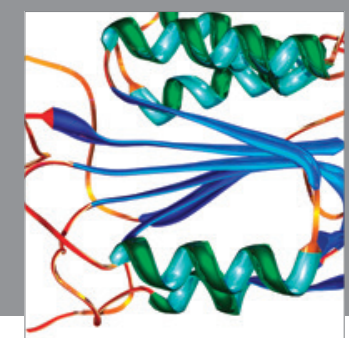

Disease Markers
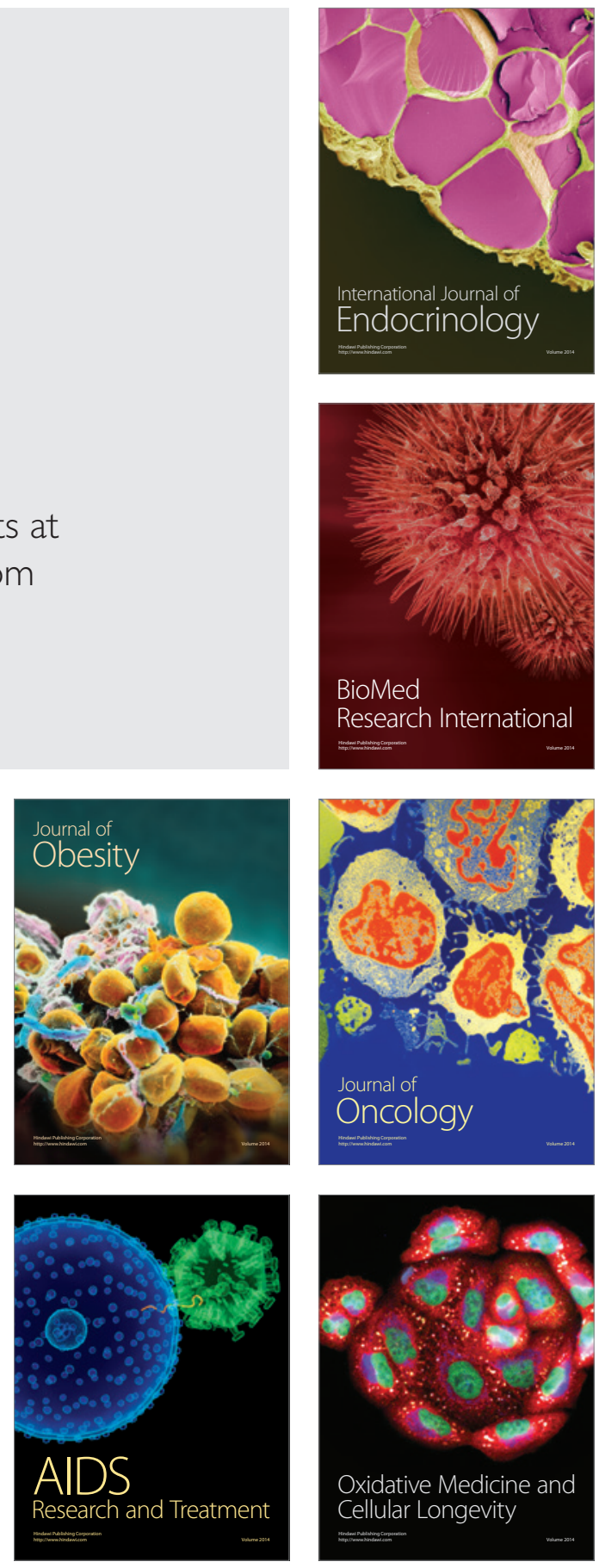